experimental test drug groups (i.e., groups III and IV) with p-value of less than 0.05 . In another model with electrically induced seizure test, Results were of similar nature as the chemically induced seizure model, as in both the experimental test drug groups PPAR $\sigma$ agonists+, that is, groups VII and VIII, there was a slight reduction in score of seizures, when compared with the group V (standard) with p-value of more than 0.05 .

Conclusion: Peroxisome Proliferator Activated Receptor $\sigma(p \mathrm{PAR} \sigma)$ agonists showed increase in onset with decline in duration, number, and score of seizures in chemically, as well as decline in score of seizures in electrically induced seizures in experimental laboratory animal models.

\section{A0023: Impact of Repeated Health Education Delivery on Antiepileptic Drug Adherence in People with Chronic Epilepsy in the Community \\ Karan Chouhan, ${ }^{1}$ Chirag Gupta, ${ }^{1}$ Archita Chawla, ${ }^{1}$ \\ Sandeep Kaushal, ${ }^{1}$ Gagandeep Singh, ${ }^{1}$ Namita Bansal, ${ }^{1}$ \\ Suman Sharma ${ }^{1}$ \\ ${ }^{1}$ Dayanand Medical College and Hospital, Ludhiana, Punjab, India}

Introduction: Epilepsy is a chronic disorder with long-term treatment, and adherence to antiepileptic drugs decreases with time. Repeated counseling with adherence reinforcement may prevent this adherence decay.

Objective: To study the impact of repeated health visits and patient education on adherence to antiepileptic drugs in people with chronic epilepsy in the community.

Methods: The setting of the study was provided by community care trial of home-based care delivered by primary health care workers versus routine clinic-based care. We studied the association between monthly pill counts and self-reported adherence questionnaire score with age, gender, religion, ethnic origin, education, occupation, monthly family income, and socioeconomic class. In addition, we created a dummy variable, which represented baseline scores for each subject.

Results: There was considerable inter individual variations on pill count and SRMS score. Overall, lag SRMS, religion, ethnic origin, and monthly family income determined adherence status. A steady increase in the proportion of people with appropriate pill count was observed in the home-based care group but not in the clinic-based care group.

Conclusion: Treatment adherence varies considerably between individuals and is influenced by individual behavior. However, repeated adherence reinforcement by primary health care workers may lead to improvement in adherence.

\section{A0024: EEG Changes in Autoimmune Neuronal Synaptic Encephalitis-A Case Study \\ Laxmi Khanna ${ }^{1}$, Nandini Agarwal ${ }^{1}$ \\ ${ }^{1}$ Department of Neurophysiology, Sir Ganga Ram Hospital, New Delhi, India}

Introduction: Autoimmune neuronal synaptic encephalitis is a group of antibody-mediated inflammatory diseases of the central nervous system. We have used bedside EEG recording in the early diagnosis of suspected cases of autoimmune encephalitis.
Methods: The EEG records of 15 patients $(\mathrm{M}: \mathrm{F}=10: 5)$ with suspected autoimmune encephalitis were studied. Their clinical profile, antibody studies, MRI findings, and outcome were also recorded.

Results: $-26.67 \%$ patients had periodic discharges in the EEG, 6.67\% patients had triphasic complexes, $20 \%$ patients had a generalized delta slowing with delta brushes, $20 \%$ patients had generalized epileptiform discharges, $13.72 \%$ had a nonconvulsive status epilepticus, and $13.72 \%$ had a rhythmic delta slowing in the EEG.

Conclusion: EEG is an inexpensive tool in the early diagnosis of suspected autoimmune neuronal synaptic encephalitis. The Results of an abnormal EEG will aide in the diagnosis and -rapid initiation of treatment even before other test Results become available. Often, antibody studies do not correlate with the clinical presentation and bedside EEG could be an invaluable guide in clinically suspected cases. Initiation of treatment early in the illness improves the clinical outcome and delays the long-term sequelae.

\section{A0025: Subjective Memory Complaints in Temporal Lobe Epilepsy What Contributes to an Experience of Memory Dysfunction? \\ Desai M., ${ }^{1}$ Rajeshree S., ${ }^{1}$ Sapre A., ${ }^{1}$ Kalika M.,, ${ }^{1}$ Shah U., ${ }^{1}$ Ravat $\mathrm{S} .^{1}$ \\ ${ }^{1}$ Department of Neurology, King Edward Memorial Hospital, Mumbai, Maharashtra, India}

Patients with temporal lobe epilepsy (TLE) often present with subjective memory complaints (SMC) that cause significant distress and impact their quality of daily life (Giovagnoli and Avanzini, 2000). Sometimes, these complaints do not correlate with Objective memory test (OMT) scores (Hall et al, 2009). Memory deficits are important variables in planning treatment and predicting surgical outcome. Hence, identifying factors that influence SMC helps in planning effective treatment.

Objective: To delineate predictors of subjective memory complaints.

Methods: Retrospective data analysis of 97 adult patients who had (1) left mesial TLE, (2) underwent comprehensive neuropsychological examination, (3) IQ > 70. A stepwise binary logistic regression was conducted using data from clinical history and test scores for independent variablesdemographics (age, gender, education, and occupation), intelligence, memory tests (R-AVLT, paired associates and complex figure test), seizure, medication, mood, and psychosocial factors (stigma and overprotection) as predictors of SMC.

Results: Seventy-five patients (77.31\%) reported SMC, of which one fourth (25.33\%) showed no impairment on any OMT. The regression model indicated two significant predictors of SMC, mood issues and one memory test (paired associates) score, $\chi^{2}=14.93, \mathrm{p}<0.001$.

Conclusion: The odds of reporting SMC increased with the presence of mood issues and poor performance on one memory test. Mood issues appear to be an important factor impacting memory significantly and resulting in subjective experience of problems despite no impairment in most Objective tests. It is important to elicit subjective 
complaints and address the mood issues to reduce burden of memory dysfunction in patients with epilepsy.

\section{A0026: Children (12-18 Years Age) of Women with Epilepsy Have Lower Intelligence, Attention, and Memory: Observations from the Kerala Registry of Epilepsy and Pregnancy \\ Manna Jose, ${ }^{1}$ Veena P., ${ }^{1}$ Nikita Susan Jacob, ${ }^{1}$ Sanjeev V. Thomas $^{1}$ \\ ${ }^{1}$ Department of Neurology, Sree Chitra Tirunal Institute for Medical Sciences and Technology, Trivandrum, Kerala, India}

Objective: We aimed to study the cognitive outcome of 12- to 18-year old children of women with epilepsy (WWE).

Methods: Children of WWE (12-18 years) under follow-up in Kerala Registry of Epilepsy and pregnancy $(n=86)$ were evaluated with Wisconsin Card Sorting Test (WCST), trail making test (TMT), Rey auditory verbal learning test (RAVLT), and Rey-Osterrieth complex figure test (ROCFT).

Results: There were 41 women with generalized epilepsy (47.7\%) and 45 with localization-related epilepsy (52.3\%). Antiepileptic drugs (AEDs) exposure was as follows: carbamazepine (26), valproate (21), phenytoin (five), phenobarbital (three), polytherapy (25), and six were unexposed. The full-scale IQ (FSIQ) and higher order executive functioning of children of these 86 WWE showed significant difference with AED exposure. The FSIQ mean \pm SD for different AEDs were phenobarbital: (73.6 \pm 14$)$, phenytoin: $(87.7 \pm$ 22.1), carbamazepine: (96.4 \pm 8.2 ), valproate: (93.6 \pm 13.6 ). The FSIQ for those exposed to phenobarbital was significantly ( $p=0.045$ ) lower than others, whereas those children unexposed to AEDs had high FSIQ scores (mean $=97.92, \mathrm{SD}=$ $10.72 ; p=0.028$ ). Higher order executive functioning, specifically the ability to maintain and shift set was found to be low in children exposed to phenobarbital and valproate monotherapy compared with no AED exposure $(p=0.049)$.

Conclusion: IQ and higher order executive functioning were significantly lower for 12- to 18 - year-old children of WWE exposed to different AEDs when compared with nonexposed children. Thus, antenatal AED exposure is an important predictor of low FSIQ and higher order executive functioning.

A0027: Effect of Antidepressants (Sertraline, Escitalopram) in Combination with Antiepileptic Drugs (Sodium Valproate, Levetiracetam) on Seizures, Cognitive Impairment, and Oxidative Stress in Rats

Md. Hussain, ${ }^{1}$ Jatinder Katyal, ${ }^{1 *}$ Haroon Rashid, ${ }^{1}$ Yogendra K. Gupta'

${ }^{1}$ Neuropharmacology Laboratory, Department of Pharmacology, All India Institute of Medical Sciences, New Delhi, India

Objectives: Depression is a major comorbidity in persons with epilepsy ( $p \mathrm{WE}$ ). The effect of antidepressants sertraline (SRT) and escitalopram (ESC) in combination with sodium valproate (SV) and levetiracetam (LEV) on seizure and cognitive impairment in rats was studied.
Methods: The male Wistar rats (200-250 g) were trained for Morris water maze (MWM), passive avoidance $(p \mathrm{~A})$ and elevated plus maze (EPM) test and baseline trials conducted before subjecting to pentylenetetrazole ( $p \mathrm{TZ}$ )induced kindling. SV and LEV were used in combination with SRT and ESC to evaluate effect on seizure development, cognition, and biochemical parameters. SRT (25 mg/kg), ESC (15 mg/kg), SV (150 mg/kg), and LEV (300 mg/kg) were injected daily at the same time and seizure stimuli given every alternate day 4-h,1-h, and 30 minutes, respectively, after dosing with above drugs. On day 49, retention trials performed, rats sacrificed, and blood and brains collected for malondialdehyde (MDA) and glutathione (GSH) estimation.

Results: SRT $25 \mathrm{mg} / \mathrm{kg}$ per se showed no protective effect, with SRT and LEV 12.5\% and ESC and SV group none of the rats were kindled. In EPM, kindling increased transfer latency and drug treatments were ineffective. In PA, kindling did not alter transfer latency but SRT alone and SRT + LEV treatment decreased it as compared with baseline $(p<0.001)$. On combining SRT with LEV, opposite effects in MWM and PA were observed. There was a significant decrease in level of MDA in SRT as well as ESC + SV group as compared with normal control on day 49.

Conclusion: Use of antidepressants in PWE has to be done cautiously as their effect on seizure and cognitive impairment may vary.

\section{A0028: "Super-Focal" Cortical Resection in MRI-Negative Epilepsy using Multimodal Imaging-Guided Stereo EEG: A Case Report \\ Moksh Sethi, ${ }^{1,2}$ Patrick Carney, ${ }^{1,1,3}$ Laura K. Zugno, ${ }^{1}$ \\ John Archer, ${ }^{1,2}$ Graeme Jackson ${ }^{1,2}$ \\ ${ }^{1}$ Austin Hospital, Heidelberg, VIC, Australia \\ ${ }^{2}$ The Florey Institute of Neurosciences and Mental Health, \\ Heidelberg, VIC Australia \\ ${ }^{3}$ Eastern Health, Box Hill VIC, Australia}

Objective: To present an epilepsy surgery case report from the Comprehensive Epilepsy Program (CEP) at Austin Health (Heidelberg, Victoria, Australia). In this case, multimodal imaging-guided stereo EEG allowed for a "super-focal" resection adjacent to eloquent cortex with excellent postsurgical outcome.

Methods: Patient records at the Austin Health were reviewed. Clinical details and investigations were summarized. The patient underwent video EEG monitoring, MRI at 3 and 7 Tesla, MEG, PET, SPECT (ictal and interictal), functional-MRI (EEG-fMRI and resting state functional connectivity) prior to proceeding to invasive monitoring using stereo-EEG and electrical stimulation.

Results: All investigations were reviewed at the Comprehensive Epilepsy Program Meeting at Austin health and a plan was formulated for a very limited parietal corticectomy adjacent to eloquent cortex. The patient underwent surgery without suffering a neurological deficit, and had an excellent outcome (Engel class 1B) at 12 months.

Conclusion: In selected cases, excellent outcomes can be achieved using multimodal imaging guided stereotactically 